\title{
Analysis of the postoperative epicardial auriculogram after surgical ablation of atrial fibrillation: Risk stratification of late recurrences
}

Fernando Hornero, MD, PhD, Ignacio Rodriguez, MD, Vanesa Estevez, MD, Oscar Gil, MD, Sergio Canovas, MD, Rafael Garcia, MD, PhD, and Juan Martinez Leon, MD, PhD

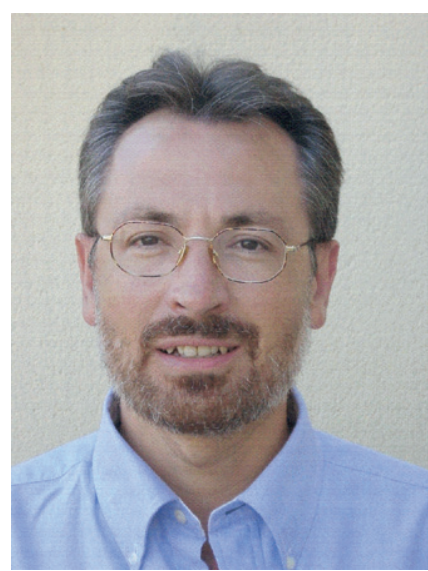

Dr Hornero
From the Department of Cardiac Surgery of Consorcio Hospital General Universitario de Valencia, Valencia University, Valencia, Spain.

Received for publication Nov 24, 2006; revisions received Jan 23, 2007; accepted for publication Jan 29, 2007.

Address for reprints: Dr Fernando Hornero, Servicio Cirugía Cardiaca, Consorcio Hospital General Universitario de Valencia, Avd. Tres Cruces s/n, 46014 Valencia, Spain (E-mail: hornero_fer@gva.es).

J Thorac Cardiovasc Surg 2007;133:1493-8

$0022-5223 / \$ 32.00$

Copyright (C) 2007 by The American Association for Thoracic Surgery

doi:10.1016/j.jtcvs.2007.01.038
Objectives: Late recurrence of atrial fibrillation frequently occurs after atrial ablation. Risk stratification for success and recurrence of the antiarrhythmic surgical procedure has not yet been established. We studied postoperative epicardial unipolar auriculograms to distinguish between high- and low-risk patients with late recurrence of atrial fibrillation.

Methods: Epicardial atrial fibrillatory activity was registered in 70 patients with surgical ablation of permanent atrial fibrillation and postoperative recurrence through the temporary wires. The atrial activation pattern was characterized in 3 groups (type I, II, and III) using Wells's criteria. The groups were homogeneous in the main clinical preoperative and surgical variables.

Results: Mean atrial frequency of postoperative atrial fibrillation recurrence showed differences between groups: $225 \pm 53 \mathrm{~ms}$ in type I, $177 \pm 21 \mathrm{~ms}$ in type II, and $150 \pm 19 \mathrm{~ms}$ in type III $(P<.01)$. At the end of the study, sinus rhythm was achieved in $80 \%$ of the subjects with type I, $87.5 \%$ with type II, and $23.8 \%$ with type III $(P<.001)$. During follow-up, late atrial fibrillation recurred in $21.7 \%$ of patients with type I, $17.4 \%$ with type II, and $64.2 \%$ with auriculogram type III. In multivariate regression analysis, the postoperative auriculogram type III was the only predictor of late atrial fibrillation recurrence (odds ratio 15.6; 95\% confidence interval, 3.2-74.7; $P<.001)$.

Conclusions: The unipolar epicardial auriculogram was able to characterize the complexity of the postoperative fibrillatory process and also to identify patients with a high risk of late recurrence. Auriculogram type III had a low success rate for the intraoperative ablation procedure. The lines of the ablation procedure facilitated organization of the auriculograms.

$\mathrm{S}$ urgical ablation procedures have proved to be an effective strategy for the treatment of atrial fibrillation (AF). Nevertheless, with the accumulation of clinical experience with intraoperative ablation, failure of the procedures and late recurrence arrhythmia have emerged as major concerns after atrial ablation. In a long-term follow-up, the postoperative sinus rhythm recovery rate is around $73.4 \%$ to $92 \%$ with different ablation energies. ${ }^{1,2}$ Late postablation recurrence of arrhythmia could be avoided if intraoperative tests were performed, as is the practice during percutaneous procedures. Although some authors have mapped AF intraoperatively and treated the sources of atrial reentries, it is unusual in normal practice to perform a guided procedure, because it is enormously complex. . $^{3,4}$ 


\author{
Abbreviations and Acronyms \\ $\mathrm{AF}=$ atrial fibrillation \\ $\mathrm{ECV}=$ electrical cardioversion \\ EKG $=$ electrocardiography \\ LA $=$ left atrium \\ $\mathrm{RA}=$ right atrium
}

TABLE 1. Baseline characteristics and preoperative echocardiographic variables of atrial sizes ( $n=70$ patients)

\begin{tabular}{|c|c|}
\hline \multicolumn{2}{|l|}{ Clinical characteristics } \\
\hline Age (y) & $62.3 \pm 11.8$ \\
\hline Male/female gender (n) & $24 / 46$ \\
\hline EKG voltage F-wave (mV) & $0.102 \pm 0.09$ \\
\hline AF long-standing $(y)$ & $6.6 \pm 5.9$ \\
\hline$<1$ & $9(12.8)$ \\
\hline $1-3$ & $12(17.1)$ \\
\hline $3-6$ & $18(25.7)$ \\
\hline $6-10$ & $13(18.5)$ \\
\hline$>10$ & $18(25.7)$ \\
\hline Antiarrhythmic drug tested & $2.3 \pm 0.7$ \\
\hline Diabetes mellitus $(\%)$ & $10(14.2)$ \\
\hline Arterial hypertension (\%) & $27(38.5)$ \\
\hline Respiratory pathology (\%) & $11(15.7)$ \\
\hline Reoperation (\%) & $8(11.4)$ \\
\hline Thromboembolic antecedent & $16(22.8)$ \\
\hline Mitral valve disease (\%) & $67(95.7)$ \\
\hline Tricuspid valve disease (\%) & $16(22.8)$ \\
\hline Aortic valve disease $(\%)$ & $26(37.1)$ \\
\hline CAD $(\%)$ & $14(20)$ \\
\hline Atrial septal defect (\%) & $1(1.4)$ \\
\hline Rheumatic etiology & $54(77.1)$ \\
\hline Degenerative & $9(12.8)$ \\
\hline Ischemic & $4(5.7)$ \\
\hline \multicolumn{2}{|l|}{ Preoperative atrial size } \\
\hline Anteroposterior LA diameter (mm) & $54.6 \pm 16.5$ \\
\hline Anteroposterior LA diameter $<45 \mathrm{~mm} \mathrm{( \% )}$ & $56(80 \%)$ \\
\hline Area $\mathrm{LA}\left(\mathrm{cm}^{2}\right)$ & $38.1 \pm 10.6$ \\
\hline Volume LA (mL) & $129.2 \pm 52.0$ \\
\hline Volume LA/surface body area & $78.7 \pm 31.7$ \\
\hline Area $\mathrm{RA}\left(\mathrm{cm}^{2}\right)$ & $21.2 \pm 5.3$ \\
\hline Volume RA (mL) & $77.4 \pm 50.2$ \\
\hline Volume RA/surface body area & $47.6 \pm 33.8$ \\
\hline
\end{tabular}

Data are given as mean $\pm S D$ or number (\%). $A F$, Atrial fibrillation; $L A$, left atrium; $R A$, right atrium; $C A D$, coronary artery disease.

(30\%), and a pattern of isthmus (pulmonary veins isolation, left mitral isthmus, line between right pulmonary veins and mitral annulus, and cavotricuspid isthmus) in 3 (18.5\%). All the ablation lines of the LA were performed from the endocardium side, and in the right atrium (RA) epicardially, with the same energy protocol in both chambers. The left atrial appendage was closed only when a thrombus was found during surgery, and this happened in 2 patients of group I.

\section{Statistical Analysis}

Continuous variables are presented as means \pm SD or proportions. The data distributed normally were analyzed by Student $t$ test, and data not distributed normally were analyzed by the Mann-Whitney $U$ test. Categorical variables are presented as numbers and percentages and compared by Fisher exact test. Multivariate logistic regression 

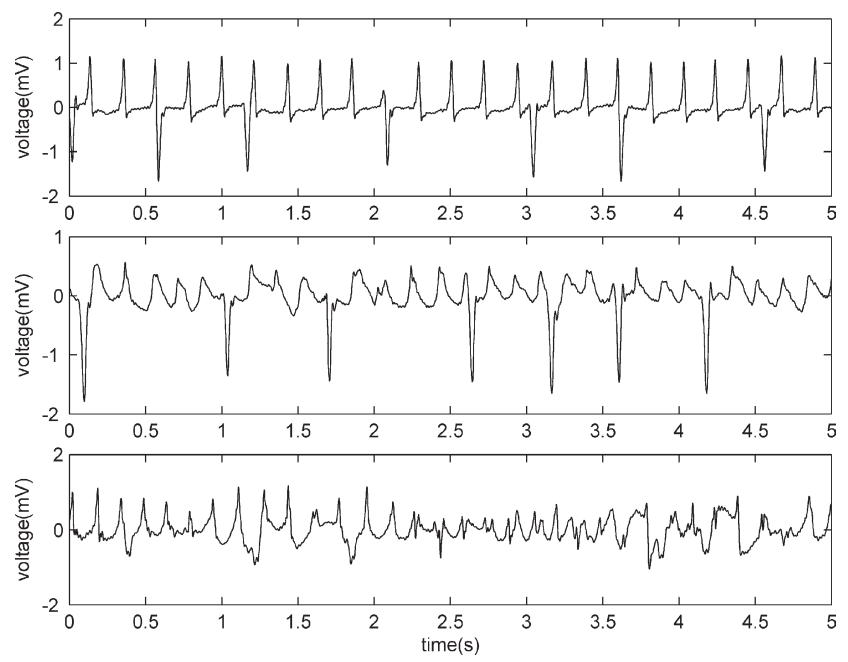

Figure 1. Epicardial auriculogram, paper speed of $25 \mathrm{~mm} / \mathrm{s}$ and amplitude of $1 \mathrm{~cm} / \mathrm{mV}$. (Top) Type I is characterized by discrete auriculogram complexes separated by an isoelectric baseline free of perturbation. (Middle) Type II is characterized by discrete auriculogram complexes but with perturbations of the baseline between complexes. (Bottom) Type III is characterized by auriculogram complexes that failed to demonstrate either discrete complexes or isoelectric baseline.

analysis was performed as conditional backward stepwise proportional hazards. Kaplan-Meier curves were generated to describe the cumulative incidence of AF recurrences for each auriculogram type during the follow-up, and the equality of the events distributions was assessed with the log-rank test. Analysis of the reproducibility of the auriculogram measurements in 2 consecutive cycles showed a variation of $1 \% \pm 5 \%$. The patients' clinical data were statistically processed by the SPSS 12.0 Software for Windows (SPSS Inc., Chicago, IL). This study has a mean follow-up of 658 days (195-2059), 197 patients/y, with 4 patients dying during the study. Two patients with type I and 2 with type III refused to complete the programmed electrical cardioversion (ECV) protocol and continued in AF. In these cases, the procedure was also considered statistically as a failure for the final results.

\section{Results}

\section{Organization of Postoperative Auriculograms} of the AF

The recorded recurrent episode of AF was morphologically classified as type I in $25(35.7 \%)$ patients, type II in 24 (34.2\%), and type III in 21 (30\%). The intraoperative mean cycle length before the ablation procedure was $161.1 \pm 21.2$ $\mathrm{ms}$, and all patients showed type III. New postoperative episodes of AF showed significant mean frequency differences between types of auriculogram: type I $225 \pm 53 \mathrm{~ms}$, type II $177 \pm 21 \mathrm{~ms}$, and type III $150 \pm 19 \mathrm{~ms}(P<.01$, between type I-II versus III). None of the preoperative and surgical variables were a prognostic factor of higher orga- nization. The mean obtained cycle length with the 3 lesion patterns was similar, and there was no difference between types of auriculogram.

\section{Clinical Profile of Patients With Hospital AF Recurrence}

The characteristics of the 3 groups of patients are presented in Table 2. The postoperative type of AF auriculogram was not related to any preoperative or surgical variables.

\section{Analysis of Results of Hospital and Early Time of Follow-up}

At discharge, the number of patients in sinus rhythm was: 9 patients with type I (36\%), 9 with type II (37.5\%), and only 3 patients with type III $(14.2 \%)$. Of these patients, only 4 with type I, 3 with type II, and none with type III kept the sinus rhythm stable and did not need ECV during the rest of the follow-up. The auriculogram type was a prognostic variable that predicted the success of the ablation procedure at the end of the follow-up in patients who were discharged in $\mathrm{AF}(P<.03)$. Eleven patients with type I $(73.3 \%), 12$ with type II (80\%), and 1 with type III (5.8\%) were discharged in AF and recovered the rhythm at the end of the follow-up.

During the early period of follow-up in patients who were discharged in $\mathrm{AF}$, the spontaneous conversion to sinus rhythm was more frequent in patients with type I and II (Table 3). Early failure of the surgical ablation procedure during this follow-up period, considered when sinus rhythm was not achieved after the protocol, happened in 1 patient of group I, 1 patient of group II, and in 6 of group III. Three patients $(4.2 \%)$ needed pacemaker implantation, 2 of group I and 1 of group II. In 3 patients of group I and in 1 of the group II, a percutaneous ablation procedure was performed to treat the recurrent AF and was only effective in 2 patients (group I). During this follow-up period, 1 patient with type I died from an unknown cause, and another with type I in permanent AF had a leg thromboembolic episode.

\section{Long-term Follow-up Results}

Late AF recurrence treated with ECV happened in 5 patients (21.7\%) with type I, 4 patients $(17.4 \%)$ with type II, and 9 patients $(64.2 \%)$ with type III. The time of the late recurrence was variable and unpredictable; most of them occurred during the first year of follow-up. In the subgroup of patients who kept stable sinus rhythm during the early follow-up stage and did not need ECV, late AF recurrence happened in $3(100 \%)$ patients with type III, 1 (25\%) with type II, and none with type I. During this later period of time, 3 patients died (1 patient with type I who refused ECV and had an intestinal embolic episode, another patient with type I who had a mechanical valve and suffered a hemorrhagic cerebral episode related to acenocumarol treatment, 
TABLE 2. Comparisons of characteristics in the three groups of auriculogram types

\begin{tabular}{|c|c|c|c|}
\hline & Type I $(n=25)$ & Type II $(n=24)$ & Type III $(n=21)$ \\
\hline Age (y) & $57.0 \pm 15.1^{*}$ & $66.6 \pm 7.7$ & $62.4 \pm 9.0$ \\
\hline AF long-standing (y) & $6.2 \pm 5.8$ & $5.6 \pm 5.4$ & $8.0 \pm 6.0$ \\
\hline EKG voltage F-wave V1 (mV) & $0.121 \pm 0.08$ & $0.113 \pm 0.071$ & $0.072 \pm 0.064$ \\
\hline Reoperation (\%) & $2(8)$ & $1(4.2)$ & $5(23.8)$ \\
\hline \multicolumn{4}{|l|}{ Structural heart disease (\%) } \\
\hline Mitral valve & $24(96)$ & $22(91.6)$ & $21(100)$ \\
\hline Tricuspid valve & $6(24)$ & $5(20.8)$ & $5(23.8)$ \\
\hline Aortic valve & $7(28)$ & $9(37.5)$ & $10(47.6)$ \\
\hline CAD & $4(16)$ & $6(25)$ & $4(19)$ \\
\hline Anteroposterior LA diameter (mm) & $51.8 \pm 9.4$ & $58.5 \pm 28.2$ & $53.8 \pm 8.5$ \\
\hline Area $L A\left(\mathrm{~cm}^{2}\right)$ & $39.0 \pm 12.9$ & $38.8 \pm 11.3$ & $36.8 \pm 9.7$ \\
\hline Volume LA (mL) & $137.1 \pm 37.3$ & $136.6 \pm 48.7$ & $116.8 \pm 54.9$ \\
\hline Volume LA/surface body area & $82.7 \pm 39.3$ & $85.5 \pm 30.9$ & $69.6 \pm 31.8$ \\
\hline Area $\mathrm{RA}\left(\mathrm{cm}^{2}\right)$ & $21.5 \pm 6.7$ & $21.6 \pm 3.3$ & $20.6 \pm 5.5$ \\
\hline Volume RA (mL) & $97.4 \pm 19.1$ & $47.4 \pm 17.8$ & $66.0 \pm 18.2$ \\
\hline Volume RA/surface body area & $60.8 \pm 6.5$ & $30.6 \pm 10.9$ & $38.3 \pm 15.6$ \\
\hline \multicolumn{4}{|l|}{ Pattern of lesions } \\
\hline \multicolumn{4}{|l|}{ Procedure } \\
\hline Maze III (\%) & $13(57)$ & $12(46)$ & $11(52)$ \\
\hline Mini-Maze (\%) & $6(26)$ & $11(42)$ & $4(19)$ \\
\hline Lineal pattern (\%) & $4(17)$ & $3(12)$ & $6(29)$ \\
\hline \multicolumn{4}{|l|}{ Ablation energies } \\
\hline Cryoablation (\%) & $6(24)$ & $6(25)$ & $5(23.8)$ \\
\hline Radiofrequency (\%) & $11(44)$ & $5(20.8)$ & $6(25)$ \\
\hline Microwaves (\%) & $7(28)$ & $13(54.1)$ & $11(52.3)$ \\
\hline
\end{tabular}

Data are given as mean \pm SD or number (\%). Auricular diameters were measured in millimeters by echocardiography; the auricular area was planimetered in the 4-chamber apical view, and auricular volume was calculated using the ellipse formula. Unipolar radiofrequency (100 W for 120 seconds, under temperature control with an upper limit of $85^{\circ} \mathrm{C}$ ) was applied with ThermaLine system (model 15907, Boston Scientific Corporation, EP Technology, San Jose, Calif), microwaves (65 W during 65 seconds) with Flex 4 system (model 15907, Guidant Scientific Corporation, EP Technology, St Paul, Minn), and cryoablation (2 minutes of ice ball, at $-70^{\circ} \mathrm{C}$ ) with Erbe system (Erbocryo $C A$, Tubingen, Germany). $A F$, Atrial fibrillation; $L A$, left atrium; $R A$, right atrium; $C A D$, coronary artery disease. ${ }^{*} P<.05$ between type I and type II.

and another patient with type II with an acute aortic dissection) and there were 2 thromboembolic episodes ( 1 with type I who died and has been mentioned before and another patient with type III with a leg embolism during a paroxysmal AF episode).

At the end of the follow-up, for $65.7 \%$ of the patients who had immediate postoperative recurrence of $\mathrm{AF}$, the surgical ablation procedure was successful and sinus rhythm returned, 20 patients $(80 \%)$ with postoperative AF of type I, 21 patients $(87.5 \%)$ of type II, and 5 patients $(23.8 \%)$ of type III. Ablation of the AF was statistically superior among patients type I to II versus type III $(P<.001)$; there were no differences between types I and II. The mean cycle length of the auriculograms of the patients with sinus rhythm was $195.5 \pm 50.5$ $\mathrm{ms}$ versus $165.7 \pm 30.9 \mathrm{~ms}$ in patients with recurrent $\mathrm{AF}$ $(P<.03)$. At the end of the study, the antiarrhythmic treatment could be suspended in 52\%, 50\%, and $14.2 \%$ and anticoagulation in $24 \%, 29.1 \%$, and $4.7 \%$ of patients type I, II, and III, respectively.

\section{Prediction of Transition to Sinus Rhythm From Postoperative AF Recurrence}

A multivariate logistic regression analysis was carried out. Auriculogram type III in the postoperative AF recurrence was the only predictor of a new AF event (odds ratio 15.64; 95\% confidence interval: 3.2-74.7; $P<.001$; Table 4 ). To assess whether the type of atrial electrogram can be used to predict sinus rhythm, a Kaplan-Meier analysis was carried out (Figure 2). The transition to sinus rhythm was clearly more frequent in patients with type I or II $(P<.003)$.

\section{Discussion}

The main results of the present study were as follows: 1) Termination of AF by a surgical ablation procedure seems to be associated with an initially higher organization of auriculograms; the greater the abnormal morphology of atrial electrograms from postoperative $\mathrm{AF}$, the greater the likelihood that the postoperative rhythm will again develop into AF. 2) The type III group should be considered as having ineffective ablation procedure with a high risk of $\mathrm{AF}$ 
TABLE 3. Evolution of the rhythm after the postoperative protocol based on the auriculogram type

\begin{tabular}{lrrr}
\hline & & \multicolumn{2}{c}{ Final rhythm } \\
\cline { 3 - 4 } Postoperative protocol & $\mathbf{n}$ & SR & AF \\
\hline Type I & & & \\
$\quad$ Spontaneous cardioversion & 8 & 8 & 0 \\
$\quad$ Effective ECV & 10 & 8 & 2 \\
$\quad$ Ineffective ECV & 2 & 1 & 1 \\
Type II & & & \\
$\quad$ Spontaneous cardioversion & 7 & 6 & 1 \\
$\quad$ Effective ECV & 13 & 12 & 1 \\
$\quad$ Ineffective ECV & 1 & 0 & 1 \\
Type III & & & \\
$\quad$ Spontaneous cardioversion & 3 & 2 & 1 \\
$\quad$ Effective ECV & 11 & 3 & 9 \\
Ineffective ECV & 6 & 0 & 6
\end{tabular}

Values are provided as number of patients. $S R$, Sinus rhythm; $A F$, atrial fibrillation; $E C V$, electrical cardioversion.

TABLE 4. Independent predictors of atrial fibrillation recurrence

\begin{tabular}{lcll}
\hline & Odds ratio & \multicolumn{1}{c}{$\mathbf{9 5 \%}$ CI } & P value \\
\hline Gender & 1.743 & $0.371-8.2$ & .48 \\
Age (y) & 1.063 & $0.975-1.158$ & .166 \\
AF long-standing & 1.058 & $0.928-1.207$ & .399 \\
Size LA (anteroposterior) & 0.995 & $0.949-1.043$ & .826 \\
Auriculogram type III & 15.65 & $3.275-74.78$ & .001
\end{tabular}

$L A$, Left atrium.

recurrence, for whom drug anticoagulation-antiarrhythmic therapy withdrawal should therefore not be recommended. 3) Types I and II could be considered as low-risk groups, having a lower incidence of AF recurrence during the follow-up time. 4) Unipolar epicardial auriculogram characteristics could be a noninvasive surgical marker for distinguishing between high- and low-risk patients.

At the present time, intraoperative ablation is a widely used method of surgical therapy for this type of arrhythmia. However, the recurrence rate of $\mathrm{AF}$ is the most frequent problem of the procedure. Khargi et $\mathrm{al}^{1}$ reviewed 48 clinical studies with long-term follow-up results and reported that the postoperative sinus rhythm recovery rate was $78.3 \%$ (21.7\% recurrence) with different ablation energies. A recent meta-analysis, based on 69 surgical ablation studies, reports rates of freedom from late AF as between $92 \%$ and $73.4 \% .^{2}$ Various factors have been implicated in the outcome of the surgical ablation process, such as pattern of lesion, atrial size, long-standing $\mathrm{AF}$, age of the patient, among others. ${ }^{2,7,8}$ Benussi et $\mathrm{al}^{9}$ considered patient age and early postoperative arrhythmias as the only factors that

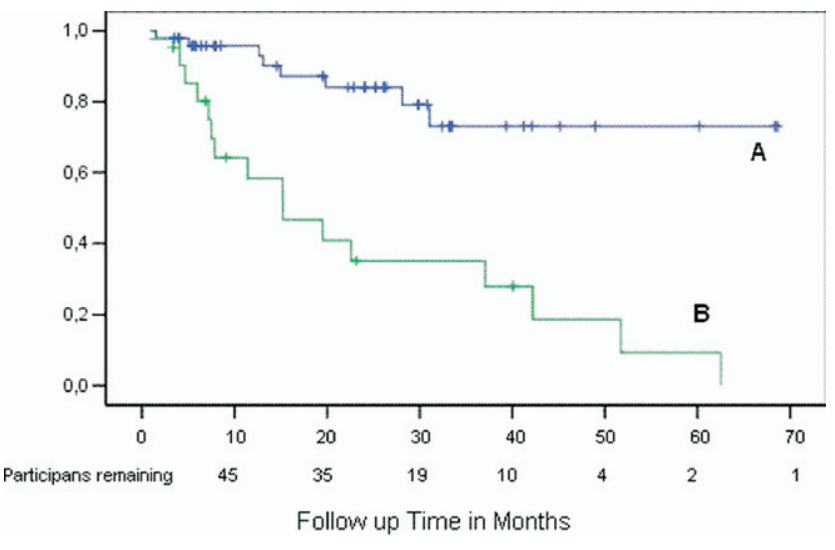

Figure 2. Kaplan-Meier actuarial curves for atrial fibrillation recurrence events based on postoperative type of auriculogram: Group A, patients with atrial electrogram types I and II $(n=49)$; Group B, patients with type III $(\mathbf{n}=21)$.

increased the risk of recurring $\mathrm{AF}$ and found 1-year freedom from AF recurrence was significantly lower in patients experiencing in-hospital postoperative arrhythmias, 67\% versus $90 \%$. These data are similar to our results of $65.7 \%$ sinus rhythm in patients with early postoperative AF recurrence. In this subgroup of patients with a postoperative recurrence episode of AF, we were looking for significant preoperative risk factors, although no variables surfaced as statistically significant predictors. Only the postoperative auriculogram type was a good predictor of late $\mathrm{AF}$ recurrence.

Surprisingly, in this era of catheter ablation and detailed endocardial mapping, only a few clinical studies have addressed the intraoperative atrial electrogram morphology and analysis of the reentries. ${ }^{4,10}$ In catheter ablation procedures, some studies have shown that successful elimination of AF was associated with a higher organization index of the auriculograms. ${ }^{11,12}$ Our study shows a process of postoperative organization of the auriculograms that is probably related to the ablation lines and the exclusion of pulmonary veins. The mean cycle length of the postoperative auriculograms of the patients who recover sinus rhythm was significantly longer than in patients who are persistently in $\mathrm{AF}$ and is related to the termination of $\mathrm{AF}$ during the follow-up. One underlying question is: how much ablation is enough, or too much if we achieve atrial organization? These epicardial EKG recordings could possibly be used to guide the surgical ablation procedure and improve the success of the technique. However, this is only a hypothesis that will need to be tested in the future.

AF can be characterized from epicardial records. ${ }^{13,14}$ In our study we did not perform an intra- or postoperative endocardial mapping study, and therefore we cannot cor- 
relate the epicardial morphology of the postoperative $\mathrm{AF}$ recurrence with the real electrophysiology substrate. Therefore, it should be emphasized that our visual classification of the AF based on epicardial unipolar auriculogram recordings does not identify the arrhythmia mechanism per se. However, we believe that with this postoperative information we can improve our follow-up protocols and make better clinical decisions for individual patients, for example, by withdrawing antiarrthymic/anticoagulation treatments. Our data suggest that the type III group should be considered as having a high risk of $\mathrm{AF}$ recurrence, and in this case, it is therefore not recommended to withdraw drug anticoagulation-antiarrhythmic therapy. Type III is probably a predictor of late failure of the ablation procedure. Moreover, patients with recurrent episodes of type I or II have a good clinical prognosis and should be recommended to repeat electrical or pharmacology cardioversion or catheter ablation before considering the procedure unsuccessful, as these maneuvers are usually successful.

Our study has several limitations. First, the number of patients in the present study was quite small. Second, we performed the auriculogram only in the daytime. It is known that in these patients other episodes can occur that may be morphologically different. Third, EKG activity may be influenced by autonomic nervous system activity at the time of EKG acquisition. Fourth, postoperative antiarrhythmic treatment reduced the mean and variability of AF frequency over time from the start of drug administration. Finally, this study is a retrospective study based on our preoperative protocol, which will differ from those of other clinical communities.

In summary, unipolar auriculogram recording may characterize the complexity of postoperative fibrillatory waves and be useful for anticipating the later clinical results of the surgical AF ablation process. It therefore could be useful in providing more precise management of drug therapy throughout the follow-up period.

\section{References}

1. Khargi K, Hutten B, Lemke B, Deneke T. Surgical treatment of atrial fibrillation; a systematic review. Eur J Cardiothorac Surg. 2005;27: 258-65.

2. Barnett SD, Ad N. Surgical ablation as treatment for the elimination of atrial fibrillation: a meta-analysis. J Thorac Cardiovasc Surg. 2006; 131:1029-35

3. Harada A, Konishi Y, Fukata M, Higuchi K, Sugimoto T, Sasaki K. Intraoperative map guided operation for atrial fibrillation due to mitral valve disease. Ann Thorac Surg. 2000;69:446-51.

4. Cox JL, Canavan TE, Schuessler RB, Cain ME, Lindsay BD, Stone C, et al. The surgical treatment of atrial fibrillation. II. Intraoperative electrophysiologic mapping and description of the electrophysiologic basis of atrial flutter and atrial fibrillation. J Thorac Cardiovasc Surg. 1991;101:406-26.
5. Wells JL, Karp RB, Kouchoukos NT, MacLean WA, James TN, Waldo AL. Characterization of atrial fibrillation in man: studies following open heart surgery. PACE. 1978;1:426-38.

6. Konings KT, Kirchhof CJ, Smeets JR, Wellens HJ, Penn OC, Allessie MA. High-density mapping of electrically induced atrial fibrillation in humans. Circulation. 1994;89:1665-80.

7. Kosakai Y. Treatment of atrial fibrillation using the maze procedure: the Japanese experience. Semin Thorac Cardiovasc Surg. 2000;12: 44-52.

8. Isobe F, Kawashima Y. The outcome and indications of the Cox maze III procedure for chronic atrial fibrillation with mitral valve disease. J Thorac Cardiovasc Surg. 1998;116:220-7.

9. Benussi S, Nascimbene S, Agricola E, Calori G, Calvi S, Caldarola A, et al. Surgical ablation of atrial fibrillation using the epicardial radiofrequency approach: mid-term results and risk analysis. Ann Thorac Surg. 2002;74:1050-7.

10. Harada A, Sasaki K, Fukushima T, Ikeshita M, Asano T, Yamauchi $\mathrm{S}$, et al. Atrial activation during chronic atrial fibrillation in patients with isolated mitral valve disease. Ann Thorac Surg. 1996;61:10411.

11. Gaita F, Riccardi R, Calo L, Scaglione M, Garberoglio L, Antolini R, et al. Atrial mapping and radiofrequency catheter ablation in patients with idiopathic atrial fibrillation. Electrophysiological findings and ablation results. Circulation. 1998;97:2136-45.

12. Takahashi Y, Sanders P, Jais P, Hocini M, Dubois R, Rotter M, et al. Organization of frequency spectra of atrial fibrillation: Relevance to radiofrequency catheter ablation. J Cardiovasc Electrophysiol. 2006; 17:382-8.

13. Holm M, Pehrson S, Ingemansson $M$, Sornmo $L$, Jahansson $R$, Sandhall L, et al. Non-invasive assessment of the atrial cycle length during atrial fibrillation in man: introducing, validating and illustrating a new ECG method. Cardiovasc Res. 1998;38:69-81.

14. Raine D, Langley P, Murray A, Dunuwille A, Bourke J. Surface atrial frequency analysis in patients with atrial fibrillation: a tool for evaluating the effects of intervention. J Cardiovasc Electrophysiol. 2004; 15:1021-6.

\section{Appendix: Postoperative Protocol}

At the time of undergoing surgery, all the patients were receiving antiarrhythmic treatment, mainly digoxin and amiodarone, which were not withdrawn for the procedure. Dual-chamber DDD stimulation was performed for 48 hours after surgery. Amiodarone was initiated in the immediate postoperative period $(1200 \mathrm{mg} / \mathrm{d}$ intravenously for 48 hours), continued for at least 3 months by oral administration $(200 \mathrm{mg} / \mathrm{d})$, or with sotalol in case of contraindication $(80-160 \mathrm{mg} / \mathrm{d})$. Anticoagulation was suspended after 6 months in those patients without mechanical prosthesis and effective $\mathrm{ECHO}$ auricular contraction (mitral A/E ratio more than 0.25 ). Subsequently, the antiarrhythmic therapy was discontinued in those cases that had recovered sinus rhythm.

During intrahospital time, postoperative AF recurrence was not electrically cardioverted except in cases that showed hemodynamic intolerance of the arrhythmia. Programmed ECV was performed between the first and third postoperative months. During the late follow-up, new persistent/permanent AF or flutter episodes were always treated with ECV, with a maximum of 2 new attempts, the surgical ablation process being then considered as ineffective and the AF as permanent. When the ECV was ineffective and EKG showed an F-wave with a high course $(>2 \mathrm{mV})$ and the patient accepted further ablation treatment, a percutaneous procedure was considered. Postoperative controls during the late follow-up were carried out after 3 and 6 months and annually with an EKG. 